\title{
Study And Investigation of Silicon Extended Source Vertical Double Gate Tunnel Transistor For Analog/RF Performance
}

omendra Kumar singh ( $\nabla$ omendra01@nitdelhi.ac.in )

National Institute of Technology Delhi

D Vaithiyanathan

National Institute of Technology Delhi

Baljit Kaur

National Institute of Technology Delhi

\section{Research Article}

Keywords: Band-to-Band Tunneling (BTBT), Subthreshold Swing (SS), Tunnel Field-Effect Transistor (TFET), Analog/RF performance, Cutoff Frequency, Energy Band

Posted Date: July 23rd, 2021

DOl: https://doi.org/10.21203/rs.3.rs-695219/v1

License: (c) (1) This work is licensed under a Creative Commons Attribution 4.0 International License. Read Full License 


\title{
Study and Investigation of Silicon Extended Source Vertical Double Gate Tunnel Transistor for Analog/RF Performance
}

\author{
Omendra Kr Singh · D.Vaithiyanathan · Baljit Kaur
}

Received: date / Accepted: date

\begin{abstract}
In this paper, a Silicon Double Gate tunnel fieldeffect transistor with Extended Source (ESVDG-TFET) is disclosed while addressing the need for dc/switching and analog/RF applications using Silvaco-Atlas simulator which is used to examine and explore the performance of the proposed device. The mechanics of band-to-band tunnelling and accompanying carrier injection are used to illustrate the operation of the proposed silicon ESVDG-TFET device. The gate is designed to overlap with extended source region along with $\mathrm{N}+$ pockets and channel in order to facilitate both the lateral and vertical tunnelling. The silicon ESVDG-TFET provide lower subthreshold swing of $10.1 \mathrm{mV} /$ decade that allow higher ratio of $I_{O N} / I_{O F F} \simeq 10^{13}$ for optimized device structural parameters with threshold voltage of $0.35 \mathrm{~V}$. Moreover, peak transconductance of $800 \mathrm{uS} / \mathrm{um}$, cutoff frequency of $82 \mathrm{GHz}$, gain bandwidth product of $16.8 \mathrm{GHz}$ and transit time of $1 \mathrm{p} \mathrm{sec}$ is obtained by proposed device.
\end{abstract}

Keywords Band-to-Band Tunneling (BTBT) - Subthreshold Swing (SS) · Tunnel Field-Effect Transistor (TFET) • Analog/RF performance $\cdot$ Cutoff Frequency $\cdot$ Energy Band

O.K.Singh

Department of Electronics and Communication Engineering, National Institute of Technology Delhi, India

E-mail: Omendra01@ nitdelhi.ac.in

\section{D.Vaithiyanathan}

Department of Electronics and Communication Engineering, National Institute of Technology Delhi, India

E-mail: dvaithiyanathan@ nitdelhi.ac.in

B.Kaur

Department of Electronics and Communication Engineering, National Institute of Technology Delhi, India

E-mail: baljitkaur@nitdelhi.ac.in

\section{Introduction}

Tunnel field-effect transistors (TFETs) have been considered as an alternatives to traditional metal oxide semiconductor FETs because of the beauty of this novel transistor as it deliver steeper switching characteristics (SS is not limted to $60 \mathrm{mV} / \mathrm{dec}$ ) and has a wide scope for low power SOC design (allow voltage scaling ). The physics of TFET is based on BTBT imparting immunity against various short channel effect.[3] Although the ON-state current of the TFETs is low due to the lack of BTBT (band-to-band tunnelling) probability at the source/channel junction. The probability of tunnelling is proportional to the energy band, charge carrier effective mass, gate oxide thickness and tunnelling barrier width[1][2]. Many studies have been published in recent years to improve the TFET's performance[6] -[18] Although the majority of researchers has focused on TFETs for low-power switching applications. Many efforts are being made around the world to improve ION, including the use of: 1) a low-bandgap material in the tunneling region[5]; 2) a double-gate architecture[6]; 3)Pocket doped double gate structure[7]; 4) a high-k gate dielectric[6]; 5) hetero gate dielectric[1][8]. Low subthreshold swing (SS), low threshold voltage $(\mathrm{Vt})$ and a high $\mathrm{ON}$-state current $\left(I_{O N}\right)$ to OFF-state current $\left(I_{O F F}\right)$ ratio are all desirable performance parameters for low-power switching and analog/RF circuits including high transconductance $\left(g_{m}\right)$ and high cutoff frequency $\left(f_{T}\right)$

Various efforts are made by researchers as reported in the literature to enhance the $\mathrm{dc}$ and analog/RF performance parameters. Wei Li et al.[9] the author proposed HTG-TFET (Heterojunction TFET with a T-Shaped Gate) having following performance index $I_{\text {on }}$ as $7.02 \mathrm{~A} / \mathrm{m}$ and $\mathrm{SSavg}=44.64$ $\mathrm{mV} / \mathrm{dec}$. The author addressed the problem of miller capacitance with the help of $\mathrm{Si} / \mathrm{SiGe}$ hetero-junction with different doping composition and gate overlap structures. B. S. Reniwal et al.[10] impact of device engineering on DGT- 
FET with gate underlap and different dielectric spacer material concept used to enhance $\mathrm{dc}$ and ac characteristics for SOC application. The author has reported $g_{m}$ as $4.4 \mathrm{uS}$ and $f_{T}$ as $5 \mathrm{GHz}$ and GBP of $1.8 \mathrm{GHz}$ for UL-LKHG DG-TFET. T. Joshi et al.[11] presented a Extended source DG-TFET in which entire source in placed in the channel only by varying $S_{W}$ an improvement in DC/RF parameter is reported. SSavg of $12.24 \mathrm{mV} / \mathrm{dec}, I_{O N} / I_{O F F}$ ratio of $2.5 * 10^{12}, g_{m}$ as 238 uS/um, $f_{T}$ of $37.7 \mathrm{GHz}$ and GBP as $3.4 \mathrm{GHz}$. Jang Hyum et al.[12] reported VS-TFET with vertical channel sandwiched by doped Si facilitating perpendicular tunnelling and gradual doping profile to address $I_{A M B}, I_{O N} / I_{O F F}$ as $10^{4}$ and SSmin of $17 \mathrm{mV} / \mathrm{dec}$.In comparison to the traditional counterpart, X.Zhao et al.[13] informed an L-shaped TFET to improve the BTBT rate and $I_{O N}$. LDD combined with HGD structure to reduce ambipolarity. Shupang CHen. et al.[14] suggested a silicon T-shape TFET (TG-TFET) with an SS of $24.4 \mathrm{mV} / \mathrm{decade}$ and an $I_{O N} / I_{O F F}$ of $6.7 * 10^{10}$. The TGTFET has a $g_{m}$ of $232 \mathrm{uS} / \mathrm{m}$ and a $f_{T}$ of $11.9 \mathrm{GHz}$, as investigated by the authors.In various other reported literature, researchers proposed advantage of vertical TFET over planer structure and its fabrication ease[15]. P. Wang et al.[16] improve average SS by suppressing low electric field with epitaxial tunnel layer in TFET structure. Exhaustive use of Si/Ge epitaxial layer in the channel have been reported. [20]

In this article, extended p+ source is made to overlap with gate electrode and $2 \mathrm{~nm}$ silicon epitaxial layer to facilitate both vertical and lateral tunnelling to improve dc and ana$\log$ /RF performance. The ESVDG-TFET improves $I_{O N}$ and as a result, $I_{O N} / I_{O F F}, S S$, and $V_{t}$ significantly improve. Furthermore, double gates and scaled device dimensions allow better gate control of drain current, resulting in a higher $g_{m}$ , which improves the device's high-frequency characteristics. 2-D commercially available TCAD tool, Silvaco ATLAS device simulator is used to examine and explore the performance of the proposed device. The proposed Silicon ESVDG-TFET achieves an $I_{O N} / I_{O F F}$ of $1 \times 10^{13}$ with an $\mathrm{SS}$ of $10.1 \mathrm{mV} /$ decade, according to simulation results. The device has a peak $g_{m}$ of $800 \mathrm{uS} / \mathrm{m}, f_{T}$ of $82 \mathrm{GHz}$ and Gain bandwidth product of $16.8 \mathrm{GHz}$ respectively.

\section{Device structure, Fabrication steps and Simulation}

The cross sectional schematic of Silicon ESVDG-TFET is shown in fig Fig. 1 and the parameters associated with proposed device are listed in the Table I as shown. The silicon ESVDG-TFET is a symmetrical typed double gate structure made up of a silicon substrate with a thickness of $10 \mathrm{~nm}$ $\left(T_{S i}\right)$. The gate electrode is made to overlap with $\mathrm{p}+$ source $10^{20} \mathrm{~cm}^{-3}$ of doping concentration and $2 \mathrm{~nm}$ silicon epitaxial layer $\left(T_{\text {epi }}\right)$ of $10^{17} \mathrm{~cm}^{-3}$ doping concentration below gate contact. The $n+$ pocket and $n$-channel region is doped with

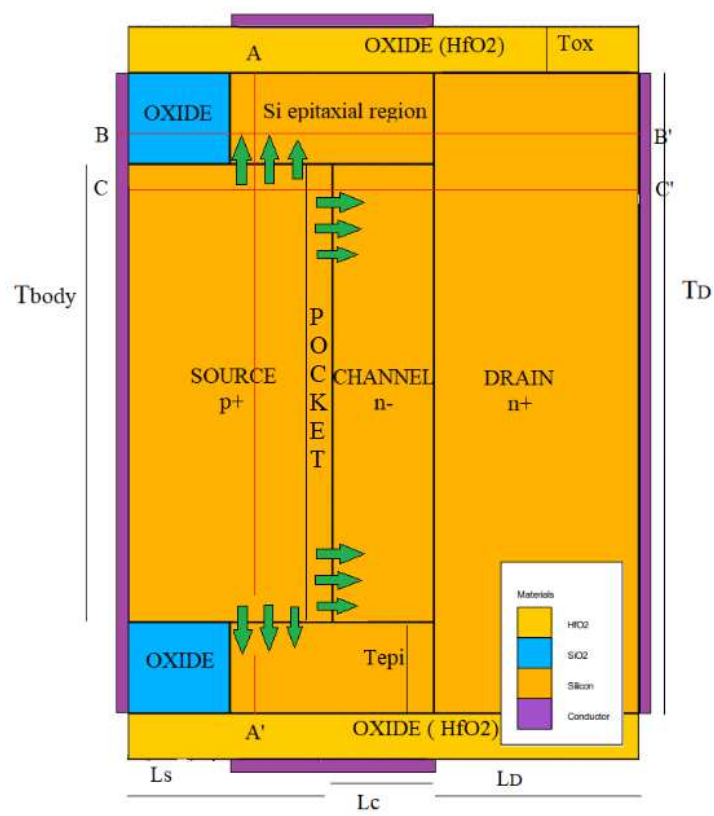

Fig. 1 Schematic of the device structure (not to be scaled).

concentration of $10^{19} \mathrm{~cm}^{-3}$ and $10^{17} \mathrm{~cm}^{-3}$ respectively $T_{\text {body }}$ and $L_{S}$ represent the height and length of the source, respectively. Similarly $L_{c}$ for channel length which is choosen to be $20 \mathrm{~nm}$. The doping concentration in the $\mathrm{n}+$ drain region is $10^{19} \mathrm{~cm}^{-3}$. A stacked gate oxide near source region (2-nm $\mathrm{SiO} 2$ and 1-nm HfO2) and as one reaches near the $2 \mathrm{~nm}$ epitaxial layer oxide thickness ( $T_{o x}$ ) of $\mathrm{HfO} 2$ reduces to $1 \mathrm{~nm}$ upto drain end with a gate work function of $4.2 \mathrm{eV}$. The proposed device has a part of source $\mathrm{p}+$ extended into channel under the gate which is differ from recently reported ESDG TFET in this entire source is grown under channel.

The proposed silicon ESVDG-TFET structure can be fabricated in the same way as reported[19][23]. In Fig. 2 the proposed ESVDG TFET's fabrication steps are depicted. The source region is defined using a photoresist mask, and then $\mathrm{P}+$ implantation is performed followed by oxidation which is used to grow 2-nm SiO2. After that, rotate the structure at right angle epitaxial growth of $2 \mathrm{~nm}$ silicon with doping type and concentration similar to the intrinsic channel is carried out[5]. In similar fashion $\mathrm{N}+$ is implanted to form drain region with defined doping concentration. In the succeeding step, oxidation is done to form gate oxide layer of 1-nm HfO2 then metallization and patterning are carried out to form the front and back gate of the ESVDG-TFET then source and drain contacts.

In order to study various physical phenomena and its effects in a TFET, relevant models are included in the ATLAS device simulator [27]. To study the recombination phenomenon Shockley-Read-Hall (SRH) with concentration depend life-time model is included. The non-local BTBT tunneling model is taken into account to analyze quantum tun- 
Table 1 Device parameter description for simulation

\begin{tabular}{|llll}
\hline Parameter & Symbol & VSiDMG[19] & ESVDG-TFET \\
\hline Source doping $\left(/ \mathrm{cm}^{3}\right)$ & $N_{A}$ & $10^{20}$ & $10^{20}$ \\
Drain doping $\left(/ \mathrm{cm}^{3}\right)$ & $N_{D}$ & $10^{18}$ & $10^{19}$ \\
Pocket doping $\left(/ \mathrm{cm}^{3}\right)$ & $N_{P}$ & - & $2 * 10^{19}$ \\
Channel doping $\left.\left(/ \mathrm{cm}^{3}\right)\right)$ & $N_{C}$ & $10^{16}$ & $10^{17}$ \\
Channel length $(\mathrm{nm})$ & $L_{C}$ & 20 & 20 \\
Source length $(\mathrm{nm})$ & $L_{S}$ & 80 & 22 \\
Gate length $(\mathrm{nm})$ & $L_{G}$ & 30 & 30 \\
Drain length $(\mathrm{nm})$ & $L_{D}$ & 50 & 15 \\
Gate oxide thickness $(\mathrm{nm})$ & $T_{O X}$ & 2 & 1 \\
Pocket thickness $(\mathrm{nm})$ & $T_{P}$ & - & 3 \\
Silicon thickness $(\mathrm{nm})$ & $T_{S i} / T_{\text {body }}$ & 10 & 10 \\
Epi-layer thickness $(\mathrm{nm})$ & $T_{e p i}$ & 2 & 2 \\
Gate work function $(\mathrm{eV})$ & $\phi_{m}$ & $4.0-4.5$ & 4.2 \\
Drain bias $(\mathrm{V})$ & $V_{D S}$ & 1.5 & 1.5 \\
\hline
\end{tabular}

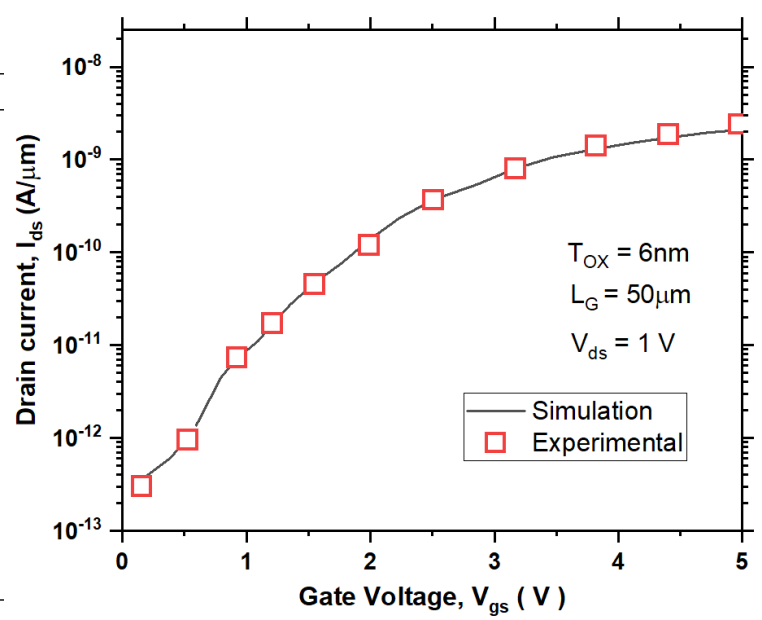

Fig. 3 Simulation model calibration using prefabricated TFET[28]
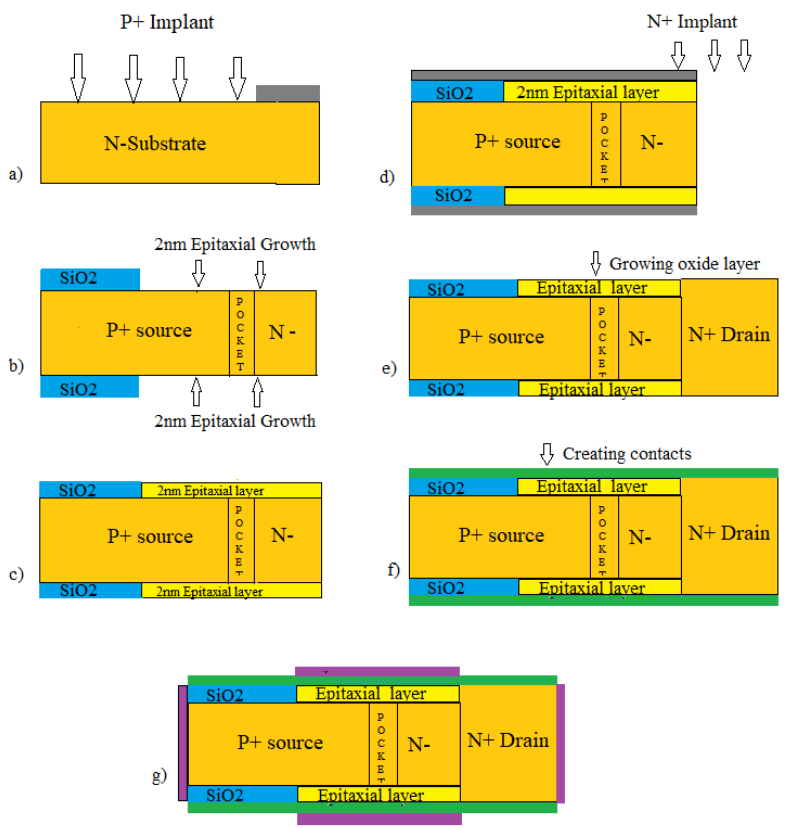

Fig. 2 Fabrication steps of proposed device structure

neling in the device. The traps and dislocations at the interface of epitaxial layer are studied using the non-local trapassisted tunneling model, which is based on the Wentzel Kramer Brillouin (WKB) transmission coefficient [1],[2]. To account for the effect of heavy doping, a band-gap narrowing model and Fermi-Dirac statistics were used. The simulation setup's accuracy is validated in the simulator by implementing a fabricated TFET [28]. We exhibit a comparison of simulated data and measured value transfer properties of a manufactured TFET. The experimental results are substantially identical to the simulated data plot as shown in Fig. 3.

\section{Results and Discussion}

\subsection{Analysis}

Using energy band diagrams, BTBT tunnelling and electric field distribution enhancement in on current can be better described with the help of Transfer characteristics within the framework.In non-local models, the most widely used method to calculate tunnelling probability is the WKB approximation based on charge carrier transmission probability (TWKB) function of tunneling barrier width is formulated as[1]

$$
T_{W K B} \propto \exp \left(-\frac{4 \sqrt{2 m^{*}} E_{g}^{3 / 2}}{3|e| \hbar\left(E_{g}+\Delta \Phi\right)} \sqrt{\frac{\varepsilon_{\mathrm{Si}}}{\varepsilon_{\mathrm{Ox}}} t_{\mathrm{OX}} t_{\mathrm{Si}}}\right)
$$

where $E_{g}$ is the bandgap, $m^{*}$ is the effective carrier mass, $\varepsilon_{o x}, t_{o x}$ are dielectric constants and oxide thickness. Also, $\varepsilon_{S i}$ and $t_{S i}$ are the silicon dielectric constants and body thickness and $\Delta \phi$ is the energy range over which tunneling can take place.

In Fig. 4 the device's transfer characteristics is presented for varying $\mathrm{Vgs}$ at $\mathrm{Vds}=1.0 \mathrm{~V}$, as indicated in plot. When energy band are not aligned at $\mathrm{Vgs}=0 \mathrm{~V}$ a weak off current flows of order $1 * 10^{-16}$. The drain current found to be independent of gate voltage in OFF-state. This region is marked by generation and recombination current (temperature dependent) dominates $I_{O F F}$. In subthreshold region that is near threshold voltage at $\mathrm{Vgs}=\mathrm{Vth}=0.35$ ( corresponding $I_{d}=$ $10^{-7}$ ) as determined by constant current method[1] and reduction in SS. Further increase in $\mathrm{Vgs} I_{d}$ is primarily governed by BTBT hence becomes less temperature-sensitive. $I_{\text {on }}$ of order $10^{-3}$ with $I_{\text {on }} / I_{\text {off }}$ ratio $10^{13}$ in case of proposed silicon ESVDG TFET which is significantly higher when compared to simulated reference device vertical $\mathrm{Si} / \mathrm{Ge}$ dual material gate TFET $I_{\text {on }} / I_{\text {off }}$ ratio $4.8 * 10^{12}$ and $I_{\text {on }} /$ $I_{\text {off }}$ ratio $4.8 * 10^{11}$. 


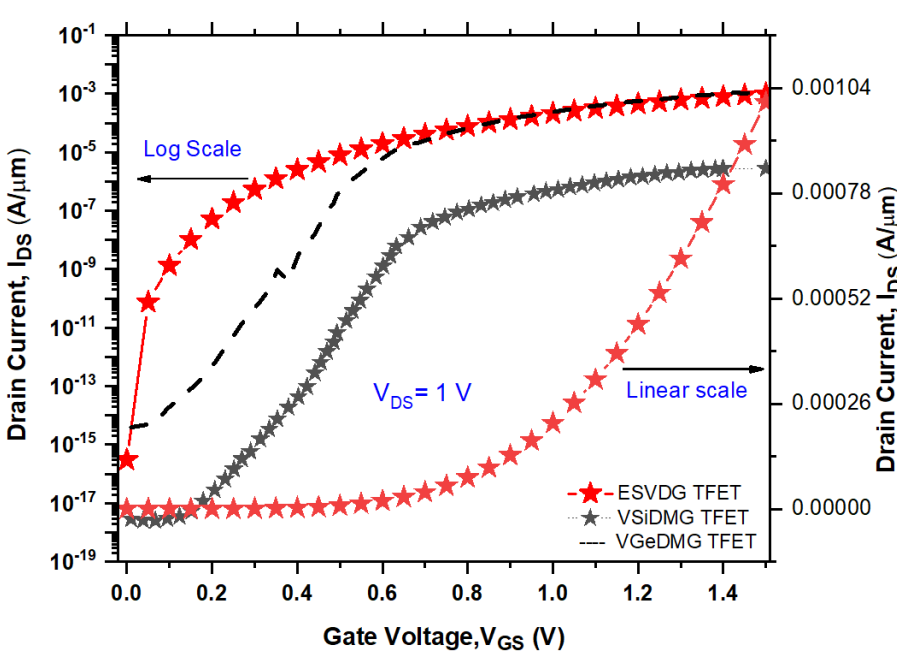

Fig. 4 Transfer characteristics curve for proposed Silicon ESVDG TFET at $\mathrm{Vds}=1 \mathrm{~V}$

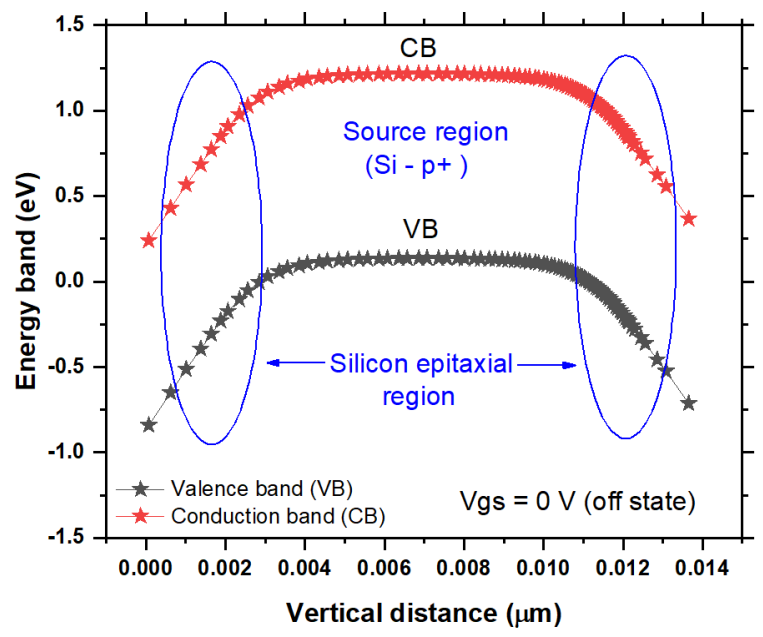

Fig. 5 Energy band plot along the cutline A-A' in OFF state at Vgs = $0 \mathrm{~V}$ and $\mathrm{Vds}=1 \mathrm{~V}$ )

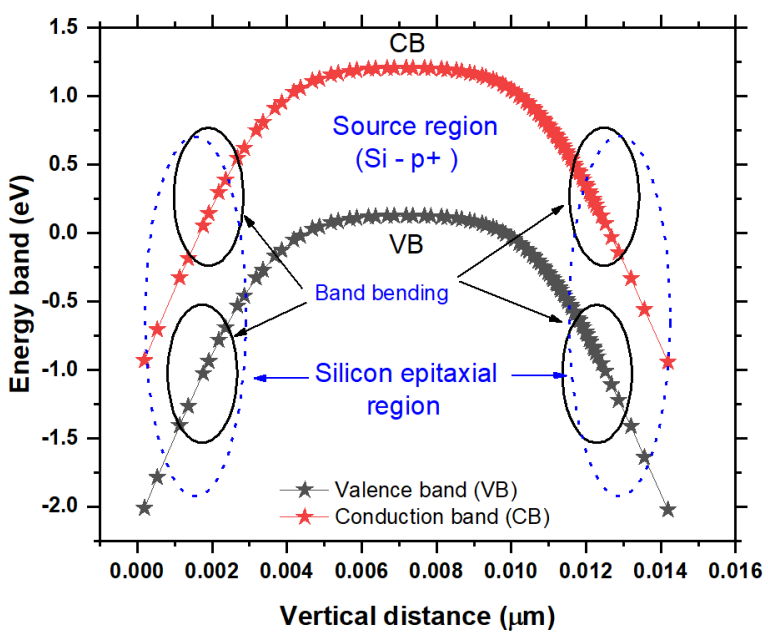

Fig. 6 Energy band plot plot along the cutline A-A' in ON state at Vgs $=1 \mathrm{~V}$ and $\mathrm{Vds}=1 \mathrm{~V}$

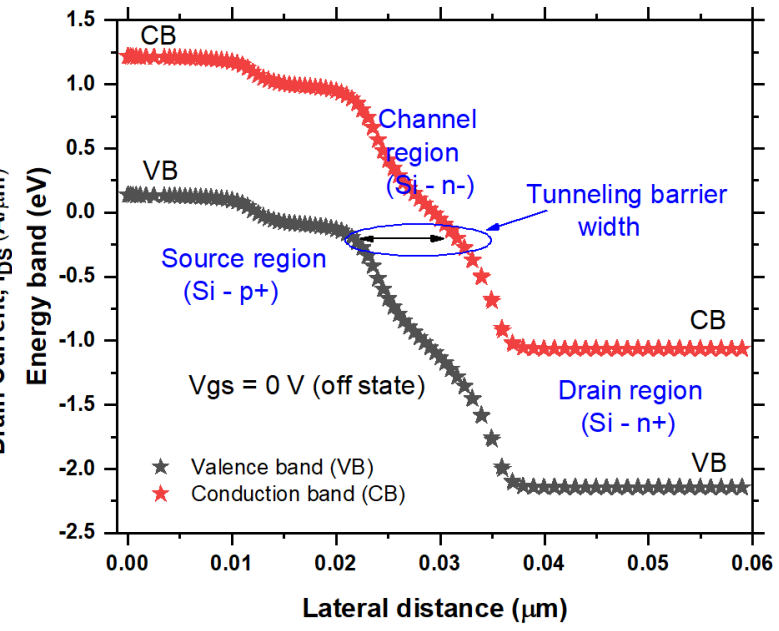

Fig. 7 Energy band plot along the cutline C-C' in OFF state at Vgs = $0 \mathrm{~V}$ and $\mathrm{Vds}=1 \mathrm{~V}$ )

Tunnelling enhances at source-channel junction and sourcesi epitaxial layer as it behave as channel for electrons tunneling vertically and then drifted toward the drain under the influence of drain voltage. In order to fully comprehend the working operation the energy band diagrams of proposed device along the cut-line AA' is displayed in Fig. 5. It is clear from figure the source region's valence band is unable to align with conduction band of Si-epitaxial layer when $\mathrm{Vgs}=0 \mathrm{~V}$, so the off-state current is very low as device is in OFF-state. Now as the gate to source voltage is increased band bending along band lowering take place and at a certain gate voltage the source region's valence band gets align with conduction band of Si-epitaxial layer. This leads to steep increases in the current. In addition increase in the gate bias leads to reduction in tunneling barrier length and further increase in the current as illustrated in Fig. 6. giving the confirmation of line tunneling.

Further, Fig. 7 and Fig. 8 illustrates the energy band diagram along cut-line $\mathrm{CC}^{\prime}$ at OFF-state $(\mathrm{Vds}=1 \mathrm{~V}, \mathrm{Vgs}=0$ $\mathrm{V})$ and at On-state ( $\mathrm{Vds}=1 \mathrm{~V}, \mathrm{Vgs}=1 \mathrm{~V})$. On increasing the gate potential energy barrier height and tunnelling width $\lambda$ decreases giving rise to point to point tunnelling. Similarly, Fig. 9 shows the energy band in ON and OFF-state when a lateral cut-line along BB' is made. It is observed a large band gap toward the left most end due to presence of $\mathrm{SiO} 2$ oxide layer. A narrow energy band of Si-epitaxial layer and drain region is present owning to silicon material. Increase in gate bias voltage leads to band lowering providing a channel for tunnelled electron.

Fig. 10 shows the variation in carrier concentration ( electron and hole ) along the cut-line C-C' as marked in the device near off state $(\mathrm{Vgs}=0 \mathrm{~V}$ and $\mathrm{Vds}=0.1 \mathrm{~V})$ and in on state $(\mathrm{Vgs}=\mathrm{Vds}=1 \mathrm{~V})$. It can be seen that large carrier concentration exist at the source-pocket-channel interface. Fig. 11 is plot of $I_{O N}$ and $I_{O F F}$ with source length. 


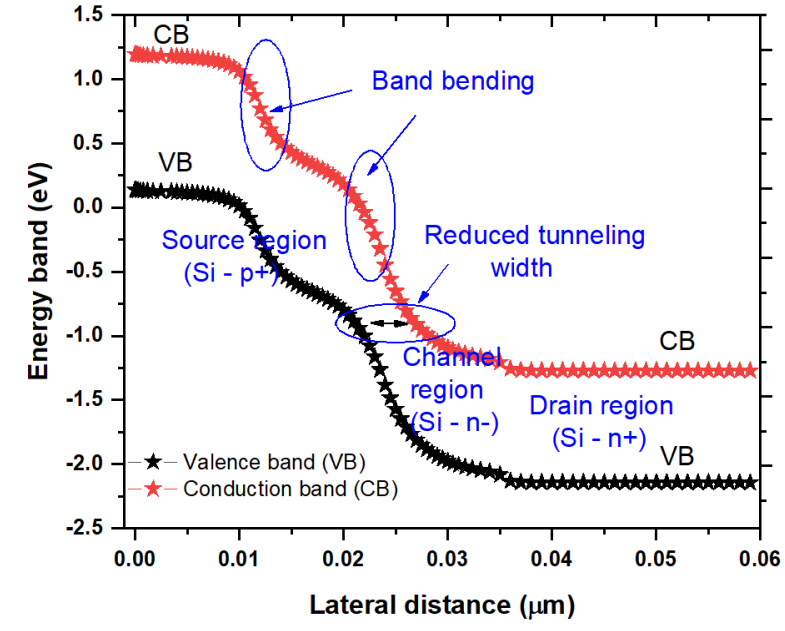

Fig. 8 Energy band plot along the cutline C-C' in ON state at $\mathrm{Vgs}=1$ $\mathrm{V}$ and $\mathrm{Vds}=1 \mathrm{~V}$ )

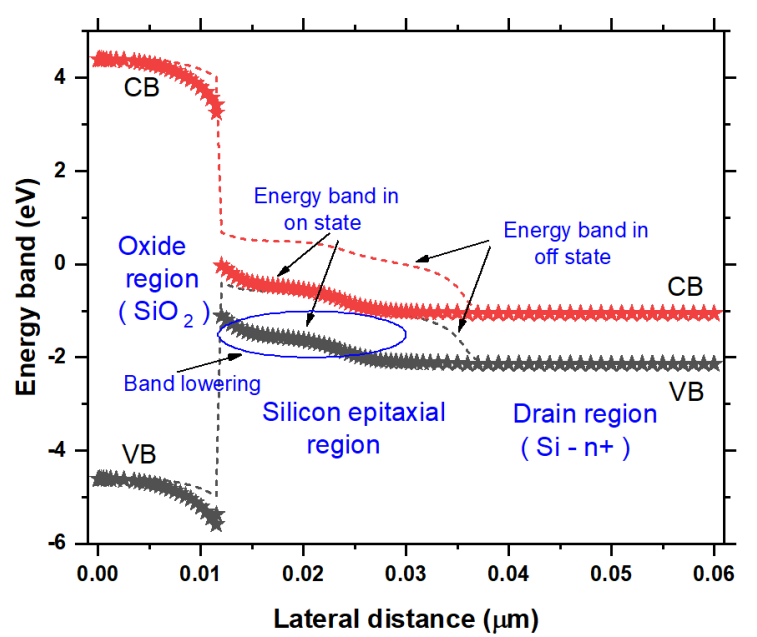

Fig. 9 Energy band plot along the cutline B-B' in OFF and ON state at varying Vgs and $\mathrm{Vds}=1 \mathrm{~V}$ )

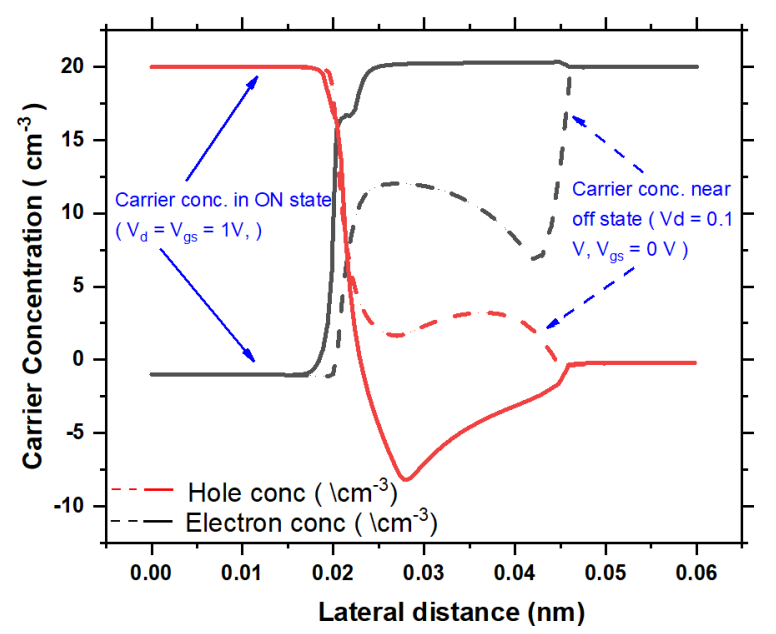

Fig. 10 Variation in carrier concentration along cutline $\mathrm{C}^{\prime} \mathrm{C}^{\prime}$ in $\mathrm{OFF}$ and $\mathrm{ON}$ state.

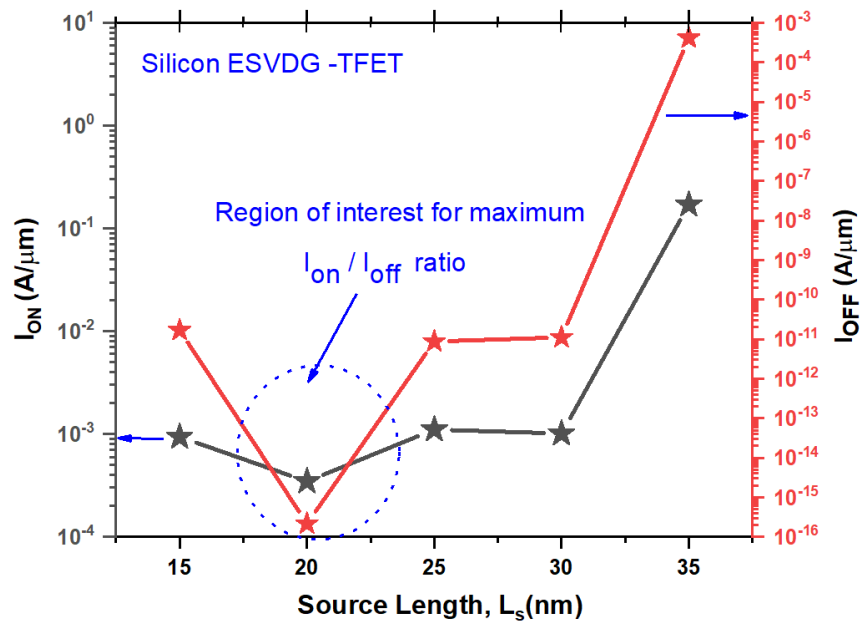

Fig. 11 Variation of Ion and Ioff current with source length.

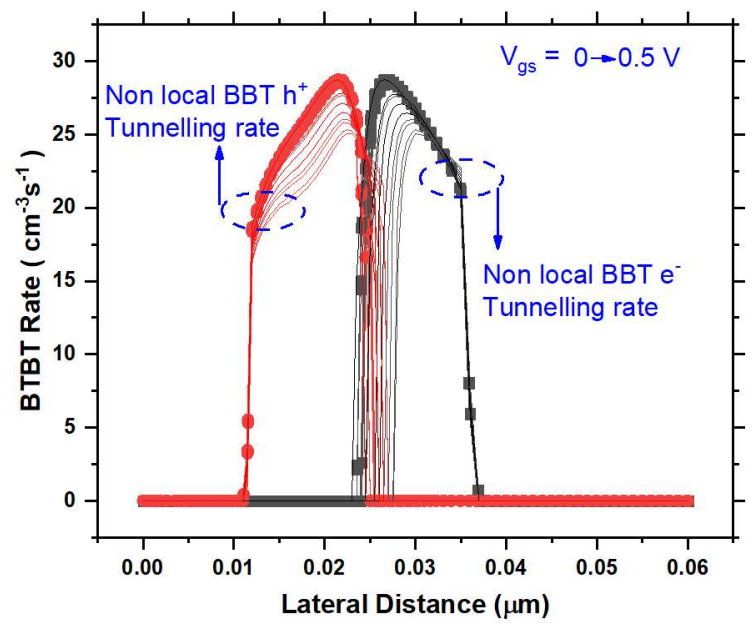

Fig. 12 Non-local BTBT tunnelling rate for varying Vgs (0 to $0.5 \mathrm{~V})$

It is seen that increase in the source length results in more tunnelling area availability, thus increase $I_{d s}$, however at the same time degradation in off current also follow thus a need for choosing optimum $L_{S}$ is necessary which is $22 \mathrm{~nm}$ for silicon ESVDG TFET at which max $I_{O N} / I_{O F F}$ is obtained while keeping $L_{G}=20 \mathrm{~nm}$. Fig. 12 illustrate non-local band to band tunnelling for hole and electron for $V_{g s}$ ranging from $0 \mathrm{~V}$ to $0.5 \mathrm{~V}$ for better understanding of proposed Silicon ES-

\section{VDG TFET.}

Fig. 13 shows the electric filed variation along the cutline $\mathrm{CC}^{\prime}$ as marked in the device. As it can be seen the peak electric field exist at the source-pocket-channel interface. It is also worth noting that the peak of the electric field of 5.15 $\mathrm{MV} / \mathrm{cm}$ at pocket doping concentration of $10^{18} \mathrm{~cm}^{-3}$ which is higher than the peak $4.6 \mathrm{MV} / \mathrm{cm}$ in the absence of pocket that to say channel doping concentration of $10^{17} \mathrm{~cm}^{-3}$. Lateral tunnelling width $\lambda$ which decrease with gate bias can be seen in energy band diagram. 


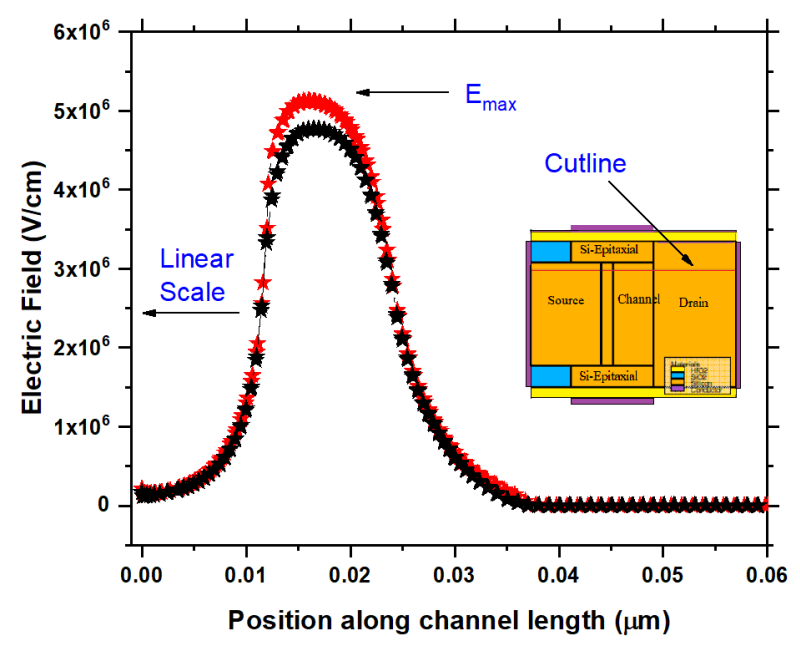

Fig. 13 Electric field plot along the cutline $\mathrm{C}^{-\mathrm{C}^{\prime}}$ at $\mathrm{Vgs}=1.5 \mathrm{~V}$ and $\mathrm{Vds}=1 \mathrm{~V}$ )

Fig. 14 depicts electric field variation along the cut-line AA' as marked in the proposed device. The maximum electric field value lies at the interface of source - silicon epitaxial of value $6.5 \mathrm{MV} / \mathrm{cm}$ for doping concentration of $10^{17}$ $\mathrm{cm}^{-3}$ which is higher than $6.3 \mathrm{MV} / \mathrm{cm}$ for doping concentration of $10^{16} \mathrm{~cm}^{-3}$. The increase in electric field with increase in doping concentration values establish direct dependency of electric field on doping thus helps in increasing the value of current within the channel doping parameter limit of International Technology Roadmap for Semiconductors(ITRS). Further, minimum electric field value of $0.02 \mathrm{MV} / \mathrm{cm}$ illustrating that mostly tunnelling take sourcechannel and epitaxial interface and least in the middle of proposed device body and significantly higher drain current flows as a result of line tunnelling. Fig. 15 depicts on current variation for different pocket doping concentration along the cut-line C-C' also schematic of device includes net doping concentration in all the region. It can be inferred as doping concentration increase electric field gets intensified near junction. Hence $I_{O N}$ current increase but this increase in doping must follow International Technology Roadmap for Semiconductors(ITRS)[33]. However in our case pocket doping of $2 * 10^{(19)}$ is chosen as improvement in current and Sub-threshold-swing is noticed.

Fig. 16 From the output characteristics it can be seen that at low Vds channel potential grows as the drain potential rises, resulting in a significant increase in current. Once drain voltage exceed gate voltage channel potential turn to be independent of drain potential thus a good saturation value off drain current $(0.325 \mathrm{~mA} / \mathrm{um}$ at $\mathrm{Vgs}=\mathrm{Vds}$ $=1.4 \mathrm{~V}$ ) for proposed silicon ESVDG TFET and value of $4.5 \mathrm{uA} / \mathrm{um}$ at $\mathrm{Vgs}=\mathrm{Vds}=1.4 \mathrm{~V}$ for simulated reference device vertical Si dual material gate TFET. Thus ESVDG TFET outperforms with other two structures.

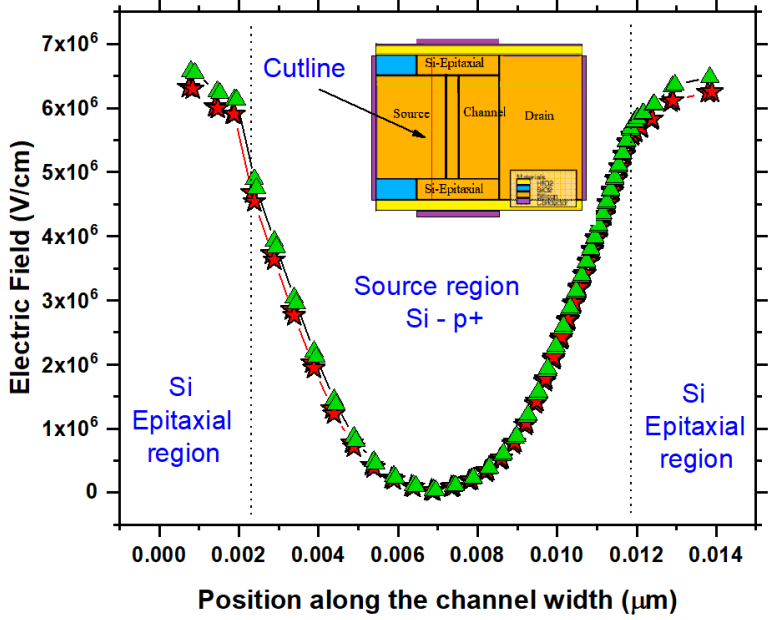

Fig. 14 Electric field plot along the cutline A-A' at $\mathrm{Vgs}=1.5 \mathrm{~V}$ and $\mathrm{Vds}=1 \mathrm{~V})$

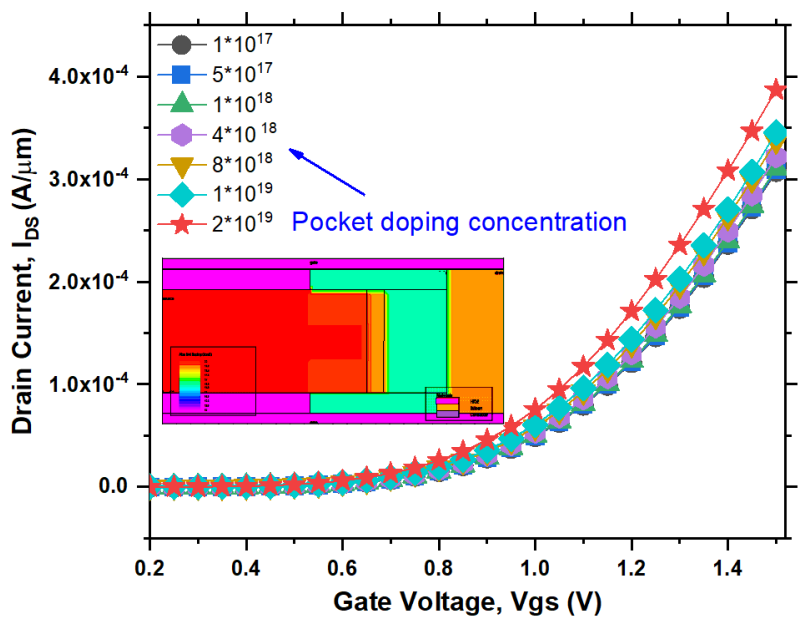

Fig. 15 Effect of change in pocket doping concentration on drain current.

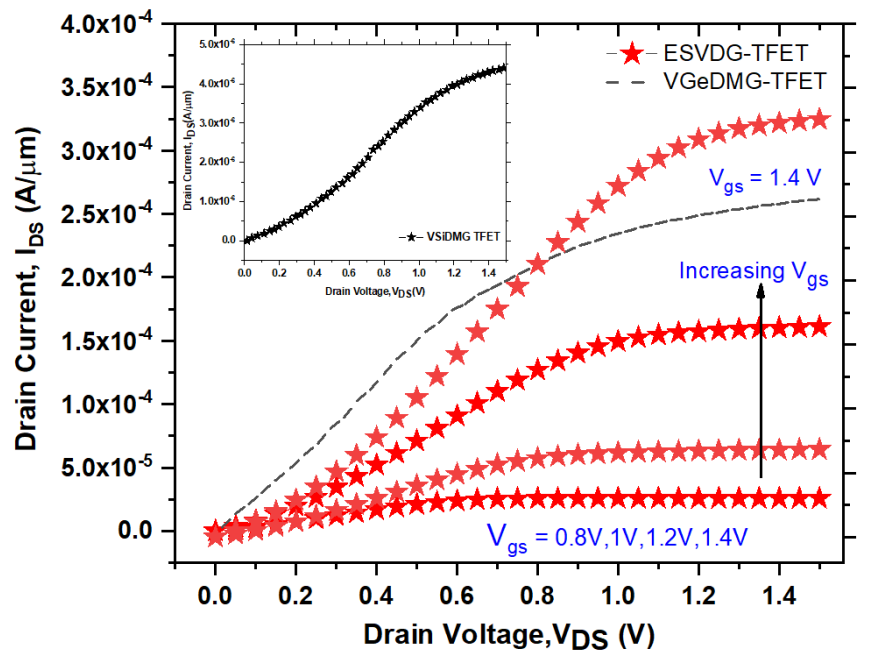

Fig. 16 Output characteristics plot of proposed silicon ESDG TFET at varying $\mathrm{Vgs}$ at $\mathrm{Vds}=1 \mathrm{~V}$ ) 


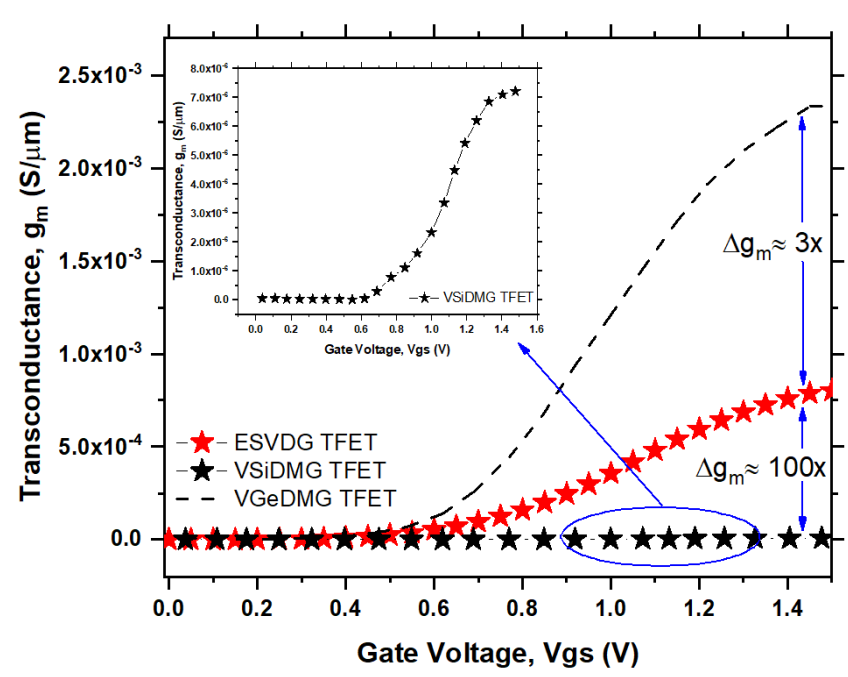

Fig. 17 Transconductance plot with varying Vgs at Vds $=1 \mathrm{~V}$

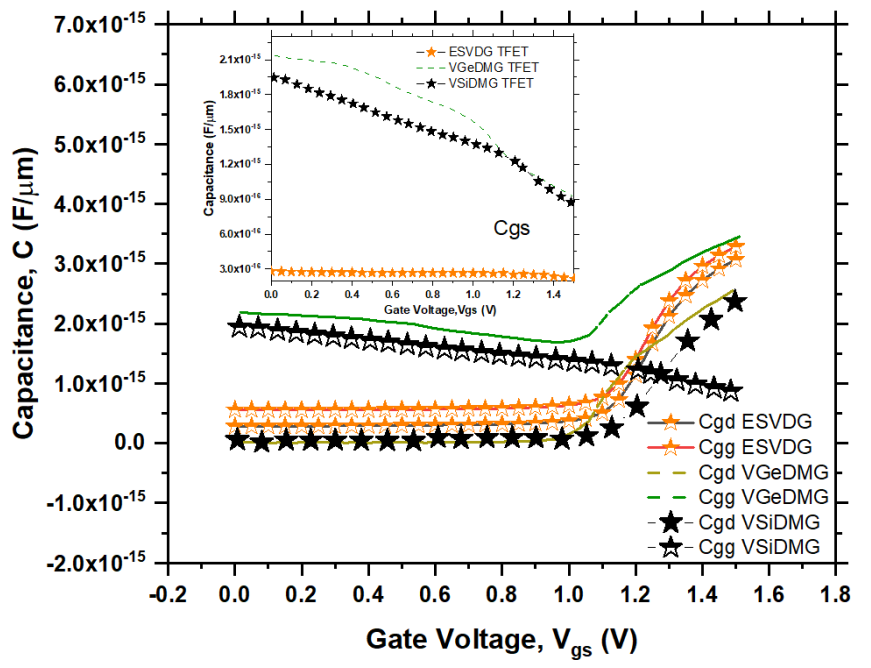

Fig. 18 Variation in capacitance's (Cgs, $\mathrm{Cgd}$ and $\mathrm{Cgg}$ ) with increasing Vgs at vds $=1 \mathrm{~V}$

Fig. 17 is a transconductance variation plot which is pivotal parameter for analog application. The amplification potential of a device is divulge by its transconductance that is more is the elevation, better is the input voltage conversion to current output as formulated in equation

$$
g_{m}=\frac{\partial I_{D}}{\partial V_{\mathrm{GS}}}
$$

It can be seen from above figure improvement in transconductance $\left(\triangle g_{m}\right)$ is in order of two decade between proposed device (silicon) and VSiDMG TFET and VGeDMG TFET shows only 3x improvement with ESVDG TFET.

\subsection{AC analysis}

The suggested device's suitability for RF applications is discussed next. The parasitic capacitance exploration is vital

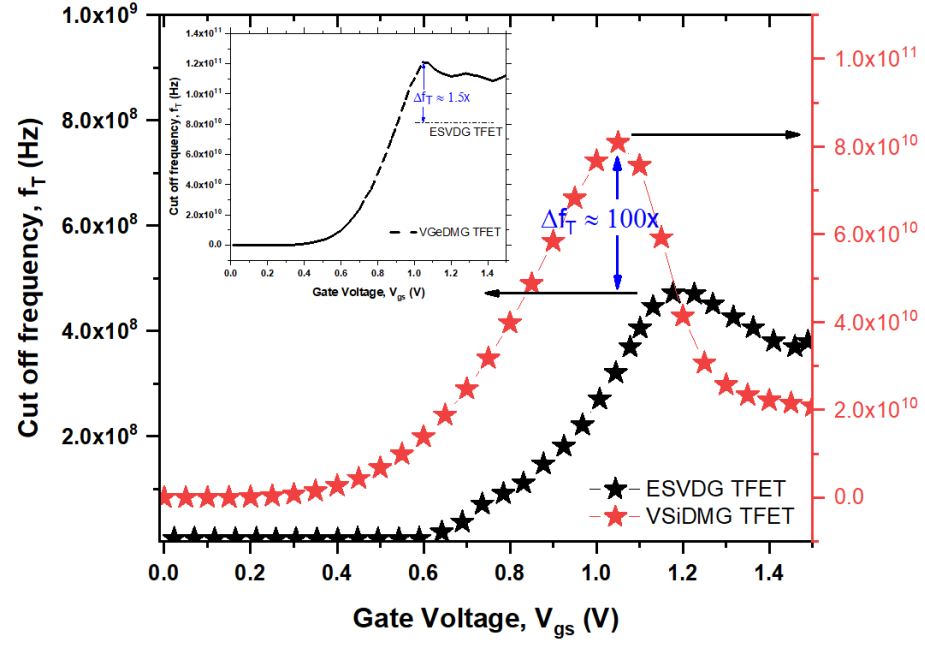

Fig. 19 Cut-off frequency $\left(f_{T}\right)$ variation with $\mathrm{Vgs}$ at $\mathrm{Vds}=1 \mathrm{~V}$

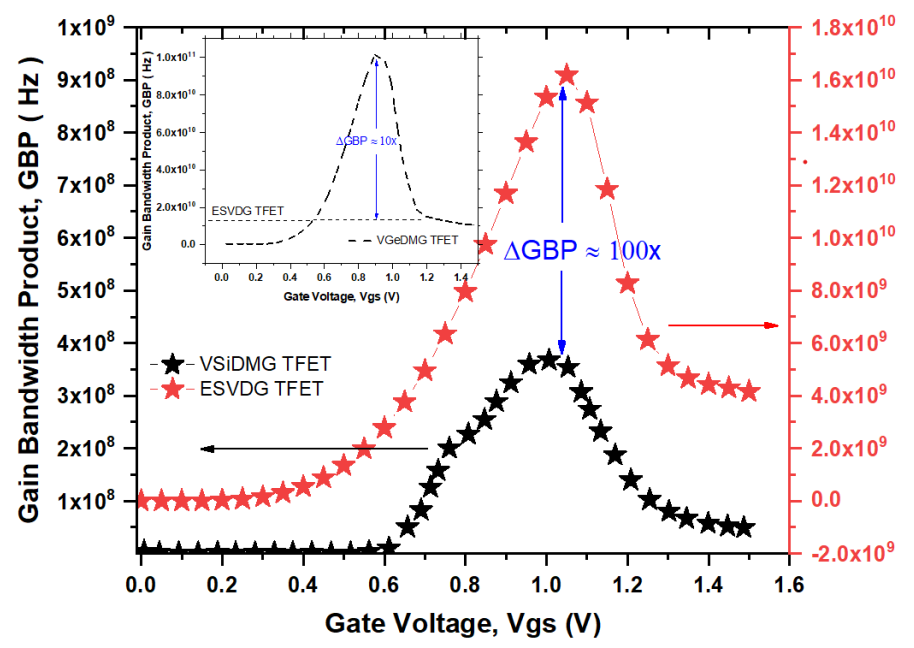

Fig. $20 \mathrm{GBP}$ variation with $\mathrm{Vgs}$ at $\mathrm{Vds}=1 \mathrm{~V}$

to study ac behaviour at high frequencies. The extraction of capacitance in the simulator is done though small signal analysis at frequency $1 \mathrm{MHz}$. Response at high frequency is mainly governed by gm and total capacitance ( $\mathrm{Cgg}$ ). As it can be seen from Fig. 18 ESVDG TFET cgs value ranges from $0.279 \mathrm{fF}$ to $0.215 \mathrm{fF}$ which can be considered to have almost a constant value. At higher voltage ( beyond $1 \mathrm{~V}$ ) slight decrements in the value of cgs occur due to reduction in gate to source coupling while cgd ranges from $0.28 \mathrm{fF}$ to $3.15 \mathrm{fF}$ and a significant increase in its value beyond $1 \mathrm{~V}$ is observed due to accumulation of more no of carrier ( electron ) at the gate interface in the channel. Total capacitance ( cgg ) is determined by additive sum of cgs and cgd. Cgg below $1 \mathrm{~V}$ is contributed by cgs and at higher voltage cgg follows cgd. It is evident from the figure, at high Vgs value ESVDG TFET shows higher Cgg value than simulated reference device vertical Si dual material gate TFET. 


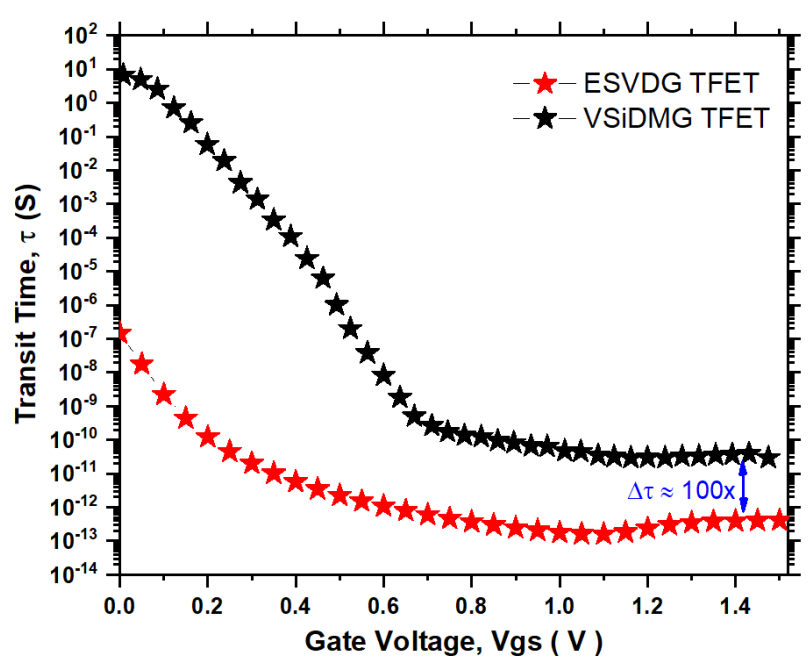

Fig. 21 Transit time variation with increasing vgs and constant Vds at $1 \mathrm{~V}$ for proposed silicon ESVDG TFET and simulated reference VSiDMG TFET

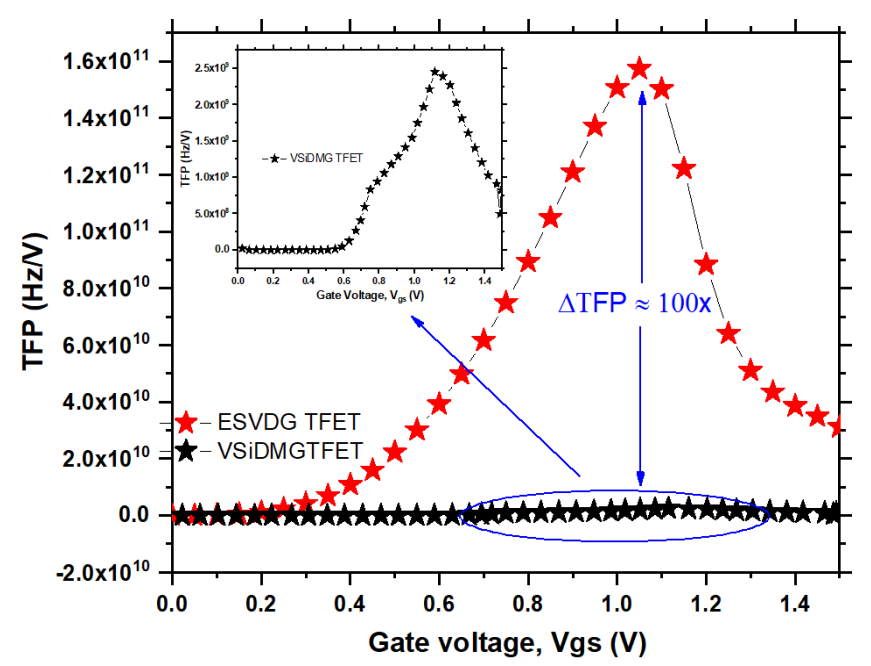

Fig. 22 Plot of TFP parameter variation with $\mathrm{Vgs}$ at $\mathrm{Vds}=1 \mathrm{~V}$

$f_{T}$, GBP and transit time, at high frequency are important parameter of concern. Higher the value of first two parameter of the device more suitable for numerous analog applications. The device cutoff frequency $\left(f_{T}\right)$ is determined by gm and Cgg which can be formulated as

$$
f_{T}=\frac{g_{m}}{2 \pi C_{g s} \sqrt{1+2 \frac{C_{g d}}{C_{g s}}}} \approx \frac{g_{m}}{2 \pi C_{g g}}
$$

From Fig. 19 it can be observed $f_{T}$ increases with Vgs due to direct dependency on gm but as soon as it attains a peak value of $82 \mathrm{GHz}$ near $1 \mathrm{~V}$, it begins to decline due to rapid increase in the value of $\mathrm{Cgg}$. However, An improvement of nearly two decade can be seen when compared with silicon ESVDG TFET and simulated reference device VSiDMG TEFT. Similarly it can be observed from Fig. 20 GBP in-
Table 2 : Comparison table for analog $/ \mathrm{RF}$ performance $\begin{array}{lllllll}\text { Parameter } & I_{o n} / I_{o f f} & \mathrm{SS}(\mathrm{mV} / \mathrm{dec}) & g_{m}(\mathrm{uA} / \mathrm{um}) & f_{T}(\mathrm{GHz}) & \mathrm{GBP}(\mathrm{GHz}) & \tau(p s e c\end{array}$ T-TFET|14] $\quad 10^{7} \quad 14.07$ VS TFET TGTFET $[9]$
VSiDMG[19] ES TFET|11| Proposed Work

$\begin{array}{cccccc}10^{7} & 14.07 & - & - & - & - \\ 10^{11} & - & 25 & 13 & - & - \\ 10^{10} & 24.4 & 232 & 11.9 & 2.3 & - \\ 10^{12} & 37 & 7.37 & 0.482 & 0.376 & 0.448 \\ 10^{12} & 12.24 & 238 & 37.7 & 3.4 & - \\ 10^{13} & 10.1 & 800 & 82 & 16.8 & 1\end{array}$

creases with Vgs due to direct dependency on gm and inversely on Cgd but as soon as it attains a peak value of $16.8 \mathrm{GHz}$ near $1 \mathrm{~V}$, it begins to decline due to rapid increase in the value of Cgd. However, An improvement of nearly two order of magnitude can be seen when compared with silicon ESVDG TFET and simulated reference device VSiDMG TEFT. The device GBP which can be formulated as

$$
G B P=\frac{g_{m}}{20 \pi\left(C_{g d}\right)}
$$

In Fig. 21 the device's transit time is presented for varying $\mathrm{Vgs}$ at $\mathrm{Vds}=1.0 \mathrm{~V}$, as indicated in plot. In general higher switching speed (lower transit time) is the desirable characteristics for a device which can be formulated as

$$
\tau=\frac{1}{20 \pi * f_{T}}
$$

At lower gate to source voltage $\tau$ seems to high due to low value cut-off frequency and decline with increase in Vgs. An overall improvement in silicon ESVDG TFET of nearly two order of magnitude can be observed when compared with simulated reference device VSiDMG TEFT. From Fig. 22 Similar observation in case of TFP parameter exist TFP expressed as $\left(g_{m} * f_{T}\right) / I_{d}$

\section{Conclusion}

The Silicon ESVDG-TFET is demonstrated with the source extended into the pocket followed by channel. The structural dimension of silicon ESDG-TFET have been optimised in order to increase line and point tunnelling. Atlas 2-D simulations are used to examine the proposed device's analog/RF performance parameters. The silicon ESVDG-TFET improves metrics like $V_{t}$, SS, $I_{o n} / I_{o f f}, g_{m}, f_{T}$, and GBP significantly. Double gates structure have superior control over the tunnelling barrier at optimum dimensions, resulting in higher $g_{m}$, improved $f_{T}$, GBP, $\tau$ and TFP of order of two decade when compared with silicon based other vertical DG-TFET. Hence, Silicon ESVDG-TFET is a better candidate for low power switching and analog/RF performance applications.

Acknowledgements In particular, I thanks Dr. D.Vaithiyanathan and Dr. Bajilt Kaur for the discussion and help in the process of writing this manuscript. 


\section{Consent for publication}

The authors have consent for publication of this manuscript.

\section{Funding}

None

\section{Author's contributions}

O.K.Singh puts forward innovative results in this manuscript, completed the simulation work and article writing - Original draft preparation. D. Vaithiyanathan and B. Kaur participated in visualization, investigation, supervision, writing - review, and editing.

\section{Competing interests}

The authors declare that they have no known competing financial interests or personal relationships that could have appeared to influence the work reported in this paper.

\section{Ethical approval and consent to participate}

The authors declare that they have consent for participation.

\section{Availability of data and materials}

Not applicable

\section{References}

1. Jagadesh Kumar Mamidala,Rajat Vishnoi and Pratyush Pandey "Tunnel Field-Effect Transistors (TFET): Modelling and Simulation" First Edition. Wiley Sons, 2017

2. S. Saurabh and M. J. Kumar, Fundamentals Tunnel Field-Effect Transistors, vol. 1. Boca Raton, FL, USA: CRC Press, Nov. 2016.

3. A. M. Ionescu and H. Riel, "Tunnel field-effect transistors as energyefficient electronic switches," Nature, vol. 479, no. 7373, pp. 329-337, Nov. 2011, https://doi.org/10.1038/nature10679

4. K. Boucart and A. M. Ionescu, "Threshold voltage in tunnel FETs: physical definition, extraction, scaling and impact on IC design," ESSDERC 2007 - 37th European Solid State Device Research Conference, Munich, Germany, 2007, pp. 299-302, doi: 10.1109/ESSDERC.2007.4430937.

5. C. Le Royer and F. Mayer, "Exhaustive experimental study of tunnel field effect transistors (TFETs): From materials to architecture," in Proc. ULIS,Mar. 2009, pp. 53-56.

6. Boucart, "Simulation of a double gate silicon tunnel FET with a High Dielectric,' Ph.D. thesis, Ecole Polytechnoique Federale de

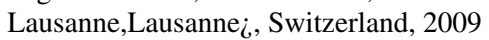

7. R. Jhaveri, V. Nagavarapu and J. C. S. Woo, 'Effect of Pocket Doping and Annealing Schemes on the Source-Pocket Tunnel FieldEffect Transistor,' in IEEE Transactions on Electron Devices, vol. 58, no. 1, pp. 80-86, Jan. 2011, doi: 10.1109/TED.2010.2089525.
8. V. D. Wangkheirakpam, B. Bhowmick and P. D. Pukhrambam, "N+ Pocket Doped Vertical TFET Based Dielectric-Modulated Biosensor Considering Non-Ideal Hybridization Issue: A Simulation Study," in IEEE Transactions on Nanotechnology, vol. 19, pp. 156-162, 2020, doi: 10.1109/TNANO.2020.2969206.

9. Chen, S., Liu, H., Wang, S. et al. Analog/RF Performance of TShape Gate Dual-Source Tunnel Field-Effect Transistor. Nanoscale Res Lett 13, 321 (2018). https://doi.org/10.1186/s11671-018-2723$\mathrm{y}$

10. V Vijayvargiya1, B S Reniwal, P Singh and S K Vishvakarma1. "Impact of device engineering on analog/RF performances of tunnel field effect transistors" Semiconductor Science and Technology, Volume 32, Number 6, May 2017

11. T. Joshi, Y. Singh and B. Singh, "Extended-Source Double-Gate Tunnel FET With Improved DC and Analog/RF Performance,' in IEEE Transactions on Electron Devices, vol. 67, no. 4, pp. 18731879, April 2020, doi: 10.1109/TED.2020.2973353.

12. J. H. Kim, S. Kim and B. Park, 'Double-Gate TFET With Vertical Channel Sandwiched by Lightly Doped Si," in IEEE Transactions on Electron Devices, vol. 66, no. 4, pp. 1656-1661, April 2019, doi: 10.1109/TED.2019.2899206.

13. Cong Li, Xiaolong Zhao, Yiqi Zhuang, Zhirui Yan, Jiaming Guo, Ru Han, Optimization of L-shaped tunneling fieldeffect transistor for ambipolar current suppression and Ana$\log$ RF performance enhancement, Superlattices and Microstructures, Volume 115, 2018, Pages 154-167, ISSN 0749-6036, https://doi.org/10.1016/j.spmi.2018.01.025.

14. Shupeng Chen, Hongxia Liu, Shulong Wang, Wei Li, Xing Wang and Lu Zhao. "Analog/RF Performance of T-Shape Gate DualSource Tunnel Field-Effect Transistor" Nanoscale Research Letters (2018) 13:321 https://doi.org/10.1186/s11671-018-2723-y

15. W. Li and J. C. S. Woo, "Vertical P-TFET With a P-Type SiGe Pocket," in IEEE Transactions on Electron Devices, vol. 67, no. 4, pp. 1480-1484, April 2020, doi: 10.1109/TED.2020.2971475

16. P. Wang and B. Tsui, "Band Engineering to Improve Average Subthreshold Swing by Suppressing Low Electric Field Band-to-Band Tunneling With Epitaxial Tunnel Layer Tunnel FET Structure," in IEEE Transactions on Nanotechnology, vol. 15, no. 1, pp. 74-79, Jan. 2016, doi: 10.1109/TNANO.2015.2501829.

17. K. Kato et al., "p-Channel TFET Operation of Bilayer Structures With Type-II Heterotunneling Junction of Oxide- and Group-IV Semiconductors," in IEEE Transactions on Electron Devices, vol. 67, no. 4, pp. 1880-1886, April 2020, doi: 10.1109/TED.2020.2975582.

18. Ashita, S. A. Loan and M. Rafat, "Insights Into the Impact of Pocket and Source Elevation in Vertical Gate Elevated Source Tunnel FET Structures," in IEEE Transactions on Electron Devices, vol. 66, no. 1, pp. 752-758, Jan. 2019, doi: 10.1109/TED.2018.2878010.

19. N. Paras, S.S. Chauhan, A novel vertical tunneling based Ge-source TFET with enhanced DC and RF characteristics for prospect low power applications, Microelectronic Engineering,Volume $\quad 217,2019,111103$,ISSN $\quad 0167-9317$, https://doi.org/10.1016/j.mee.2019.111103.

20. J. Oh et al., "Improved electrical characteristics of Ge-on-Si fieldeffect transistors with controlled Ge epitaxial layer thickness on Si substrates," IEEE Electron Device Lett., vol. 28, no. 11, pp. 1044-1046, Nov. 2007, doi: 10.1109/LED.2007.908502.

21. S. Yang, H. Lv, B. Lu, S. Yan and Y. Zhang, "A Novel Planar Architecture for Heterojunction TFETs With Improved Performance and Its Digital Application as an Inverter," in IEEE Access, vol. 8, pp. 23559-23567, 2020, doi: 10.1109/ACCESS.2020.2970502.

22. A. Mallik and A. Chattopadhyay, "Tunnel Field-Effect Transistors for Analog/Mixed-Signal System-on-Chip Applications," in IEEE Transactions on Electron Devices, vol. 59, no. 4, pp. 888-894, April 2012, doi: 10.1109/TED.2011.2181178.

23. R. Asra, M. Shrivastava, K. V. R. M. Murali, R. K. Pandey, H. Gossner and V. R. Rao, ”A Tunnel FET for $V_{D D}$ Scaling Below 0.6 
V With a CMOS-Comparable Performance," in IEEE Transactions on Electron Devices, vol. 58, no. 7, pp. 1855-1863, July 2011, doi: 10.1109/TED.2011.2140322.

24. R. Dutta, M. Rahaman, A. Guha and N. Paitya, "Study of gate source-drain overlap/gate-channel underlap in Heteojunction (50nm Ge channel) n-Double Gate TFET for different $\kappa$-spacer," 2019 International Conference on Smart Systems and Inventive Technology (ICSSIT), Tirunelveli, India, 2019, pp. 672-676, doi: 10.1109/ICSSIT46314.2019.8987903.

25. E.Datta, A.Chattopadhyay, A. Mallik and Y. Omura, "Temperature Dependence of Analog Performance, Linearity, and Harmonic Distortion for a Ge-Source Tunnel FET," in IEEE Transactions on Electron Devices, vol. 67, no. 3, pp. 810-815, March 2020, doi: 10.1109/TED.2020.2968633.

26. R. Narang, M. Saxena and M. Gupta, "Comparative Analysis of Dielectric-Modulated FET and TFET-Based Biosensor," in IEEE Transactions on Nanotechnology, vol. 14, no. 3, pp. 427-435, May 2015, doi: 10.1109/TNANO.2015.2396899.

27. Atlas User's Manual. Silvaco, Santa Clara, CA, USA, 2016.

28. P.-F. Wang, K. Hilsenbeck, Th. Nirschl, M. Oswald, Ch. Stepper, M. Weis, D. Schmitt-Landsiedel, W. Hansch, Complementary tunneling transistor for low power application, Solid-State Electronics, Volume 48,2004, doi.org/10.1016/j.sse.2004.04.006.

29. S.Singh and A.Raman, "Gate-All-Around Charge Plasma-Based Dual Material Gate-Stack Nanowire FET for Enhanced Analog Performance," in IEEE Transactions on Electron Devices, vol. 65, no. 7, pp. 3026-3032, July 2018, doi: 10.1109/TED.2018.2816898.

30. Bhagwan Ram Raad, Kaushal Nigam, Dheeraj Sharma, P.N. Kondekar, Performance investigation of bandgap, gate material work function and gate dielectric engineered TFET with device reliability improvement, Superlattices and Microstructures, Volume 94,2016, pp. 138-146, 2016,doi.org/10.1016/j.spmi.2016.04.016.

31. Menka Yadav, Anand Bulusu, Sudeb Dasgupta, Two dimensional analytical modeling for asymmetric 3T and 4T double gate tunnel FET in sub-threshold region: Potential and electric field, Microelectronics Journal, Volume 44, Issue 12,Pages 1251-1259, 2013, doi.org/10.1016/j.mejo.2013.08.011.

32. V. Vijayvargiya and S. K. Vishvakarma, "Effect of Drain Doping Profile on Double-Gate Tunnel Field-Effect Transistor and its Influence on Device RF Performance," in IEEE Transactions on Nanotechnology, vol. 13, no. 5, pp. 974-981, Sept. 2014, doi: 10.1109/TNANO.2014.2336812.

33. International Technology Roadmap for Semiconductors (ITRS) 2015 